\title{
Höhere Gewalt
}

Liebe Leserinnen, liebe Leser,

kürzlich streiften meine Augen einen Zeitungsartikel über längst vergangene Probleme und streiften dabei die Abbildung einer alten Lithographie mit einem Galgen und einer Reihe von Gehenkten. Das elektrisiert. Das Bewegendste an dem Bild: Unter den Gehenkten hing auch ein Hund. Offenbar war er mit den anderen zur Verantwortung gezogen, als schuldig befunden und aufgeknüpft worden. Ein Hund als Rechtssubjekt. Kann das sein? Kann man ein Tier zur Verantwortung ziehen, oder es vielleicht als Zeuge vernehmen, oder es gar als Kläger zulassen? Im Mittelalter und auch noch in der Zeit danach war offenbar Derartiges denkbar, Nicht-Personen als Rechtssubjekte.

Nach heutigem Rechtsverständnis können ausschließlich Personen - natürliche oder juristische - rechtsfähig sein. Nur gegen Personen schützt das Recht. Es schützt vor "Acts of Man", wie es im Angelsächsischen bezeichnet wird. Vor den sonstigen Gefahren, den "Acts of God", schützt es nicht. „Acts of Man" und "Acts of God" sind so disjunkt wie "Man" und "God". Sie schließen einander vollkommen aus, wenn man "Acts of God" wörtlich nimmt und darunter ausschließlich Naturereignisse versteht. Für Naturereignisse steht auch der Begriff "höhere Gewalt". Acts of God und höhere Gewalt sind jedoch nicht dasselbe. Es gibt höhere Gewalt, die vom Menschen kommt, z.B. Krieg; er wird nicht Gott angelastet. Acts of Man und höhere Gewalt überschneiden sich dort, wo Gefahr von der Masse Mensch ausgeht; von einer offenen ungezählten Gruppe von Personen, die miteinander anonym agieren und als Anonyme nicht zur Verantwortung gezogen werden können. Sie üben insgesamt eine höhere Gewalt aus, die von einer Nicht-Person, ihrer Masse, ausgeht. Das ist nicht auf einen Act of God zurückzuführen, sondern zu den Acts of Man zu zählen. Diese besondere höhere Gewalt kommt demnach aus einem menschlichen Bereich, der uns als solcher sehr viel vertrauter ist als der Wille Gottes. Das lässt hoffen:Wir können uns wohl gegen diese uns vertraute höhere Gewalt leichter einen Rechtsschutz vorstellen als gegen die von Gott kommenden Naturkatastrophen

In letzter Zeit häufen sich Meldungen darüber, wie jeweils Menschen von den Internet Social Utilities angereizt und zusammengeführt werden und sich dabei mit dem Hilfsmittel Social Utility spontan organisieren: Facebook Parties, Twitter Diaries, Wikileaks Veröffentlichungen, Geocoaching etc. Diese Social Utilities erschließen sich der Gesamtmasse aller Internet-Teilnehmer und stimulieren eine jeweils themenzentrierte Massenbildung. Brave New World. Meist harmlos; oft aber treten dabei die typischen, von Masse, Übermaß und Panik verursachten Schäden auf. Diese Social Utilities finden trotz - oder möglicherweise wegen - dieser Gefahren viel Zuspruch.

Das, liebe Leserinnen und Leser, berührt auch den Datenschutz. Er wurde vor vierzig Jahren entdeckt und seither zunehmend professionalisiert. Er schützt die Einzelperson vor dem Missbrauch ihrer Daten. Mehr ist nicht vorgesehen. Der Datenschutz steht aber im Gewahrsein der Öffentlichkeit - weit über den ihm zugewiesenen Bereich hinaus - für den Schutz vor allen möglichen Bedrohungen durch Informationssysteme. Doch dafür taugt er, wie sich hier zeigt, nur bedingt. Es ist ihm anzurechnen, dass er die Sicherung der Kommunikation anregt und fördert, aber er bietet keinen Schutz gegen die höhere Gewalt, die von der Nicht-Person Masse - speziell mittels Internet - ausgeht. Im Gegenteil. Ich meine: Mit dem Schutz der Anonymität leistet er dazu einen abträglichen Beitrag, wenn auch keinen entscheidenden. Weder der Datenschutz noch irgendein Recht können uns vor den Acts of God schützen, aber was schützt uns vor der Nicht-Person Masse? Darüber kann man nachdenken. Es wird aber schwierig sein. Wie soll man Nicht-Personen rechtlich beikommen? Man kann sie auch nicht henken, wie den Hund.

Mit freundlichen Grüßen, Ihr

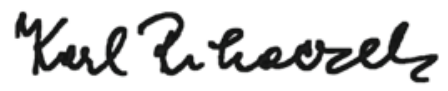

